\title{
Multipath Routing Protocol for Reducing Congestion and Delay in MANET
}

\author{
A. Pratapa Reddy \\ Assoc. Professor, Department of Computer \\ Science and Engineering , \\ Ganapathy Engineering College, Warangal, A.P.
}

\author{
N.Satyanarayana,Ph.D \\ Professor, Department of CSE \\ Nagole Institute of Science \& technology \\ Hyderabad, A.P.
}

\begin{abstract}
In this paper, a congestion aware multipath routing protocol in mobile ad hoc networks (MANET) is proposed. The paths which have better bandwidth will be initially selected. For selected paths, first the congestion status of the node is found by using the early congestion detection technique which is based on the queue status and data arrival rate of the nodes. The delay includes weighted channel delay and buffer queuing delay. The paths which have better bandwidth will be initially selected. . If the congestion status is lower than the threshold value, the packets are sent direcrly through the path. If it is more than the threshold value, a congestion aware routing is initiated. In this congestion aware routing, routes having less end-to-end delay will be selected from the backup routes, so that they have low congestion. Simulation results show that the proposed protocol reduces the end-to-end delay and increases the packet delivery ratio.
\end{abstract}

\section{Keywords}

MANET, routing protocol, congestion and delay

\section{INTRODUCTION}

\subsection{Mobile Ad hoc Networks (MANET)}

An Ad-hoc network is a set of wireless mobile nodes that forms a short-lived network any centralized administration. Mobile Ad-hoc Network (MANET) is a new model of wireless wearable devices enabling instantaneous person-to-person, person-to-machine or machine-to-person communications immediately and easily. Owing to limited transmission range of wireless network interfaces, every node transmits data to other hosts through multi-hop forwarding. Thus, every node plays the role of both router and host. MANET offers infrastructure less networking and hence nodes set up connection among them dynamically without the assistance any centralized database $[1][2][3][5]$.

An important characteristic of MANET is nonexistence of fixed infrastructure. Nodes communicate with other nodes without requiring any preexistence of infrastructure. Mobility, bandwidth, resource constraint, hidden and exposed terminal problems are some of the important characteristics to be considered while designing routing protocols for MANET. $[1][2][3][5]$

All mobile nodes are equipped with equal capabilities in MANET. A dynamic routing protocol is needed as communication links may be repaired or broken. Dynamic routing protocols require high overhead, since; it needs to discover changes in the available paths and it has to find new routing path when needed. It entirely depends on network condition. However, the efficiency of routing protocols indirectly relies on Medium Access Control (MAC) protocol. [1][2][4]

\subsection{Single Path Routing Protocol}

To route a data packet to the destination, single path routing mechanism selects a single path. Dynamic Source Routing (DSR) [4] and the Ad hoc On-demand Distance Vector (AODV) protocols are the best examples for single path routing protocol. Nevertheless, these protocols are unable of load balancing the traffic.

During route discovery process of AODV, it finds a single route between source and destination. However, it triggers route discovery process again in event of route failure in order to find an alternate path. This process experiences high delay and incurs additional resources. There is a high probability of existence of malicious node in the single path.

\subsection{Congestion and Delay in Single Path Routing}

A single path routing protocols did not fully utilize resources. It will select only one path if the path is crashing then it will select another path so that delay will be increased. Delay means packet has to reach the destination with in some time but it's taking a long time to reach the destination. The packet might take a long time to reach its destination, owing to its position in long queues, or acquires a less direct route to alleviate congestion. Congestion issues and delay issues in a single path routing are given below.

1. High overhead: In single path routing, once the primary path is broken, an alternate path discovery process is required to find another path. It costs high processing and communication effort. Alternatively, in multipath routing even the backup path is readily available; it attempts to keep up multiple paths.

2. Long delay: In critical congestion circumstances the node opts to choose a new path. However, on-demand routing protocols takes much delay to discover a new path.

3. Many Packet Losses: Most of the congestion control solutions control the congestion either by reducing data sending rate or by dropping data packets at the intermediate nodes. But, both the solutions lead to extensive packet loss [10][11] [15] [16].

\subsection{Multipath Routing Protocol}

Multipath routing means establish a multiple routes in between the source and destination. Due to the multipath routing the source nodes are capable of maintaining connections even if one route failure occurs during fault tolerance. If multipath routing protocols are used, the transmission failures and the delay times caused by route disconnection can be reduced.

In view of performance criterions such as delay, reliability, overhead, the multipath routing protocols are largely classified into five categories as minimum overhead multipath routing protocols, delay aware multipath routing protocols, reliable 
multipath routing protocols, energy efficient multipath routing protocols and hybrid multipath routing protocols [6][7].

In multipath routing protocols, the primary and backup paths are differed, when a failure happens in non-disjoint and linkdisjoint paths. Unique paths are used by both primary and backup paths in case of node-disjoint paths. The multipath routing avails instantaneous backup paths upon path failure or disconnection. The route discovery process of multipath routing considers node- disjoint, link disjoint or non-disjoint routes. Existence of mutual nodes along the path is described as nondisjoint paths, otherwise the path is referred as disjoint path [8] [9] [10].

\subsubsection{Advantages of Multipath Routing}

Fault-tolerance, load balancing and higher aggregate bandwidth are some of the benefits of multipath routing. Load balancing is accomplished by distributing the traffic among multiple paths.

Limited bandwidth is a major drawback of single path routing, where it may not offer adequate bandwidth for a connection. Since, data is routed simultaneously among multiple paths in multipath routing, the cumulative bandwidth may suit bandwidth requirement of an application [12]

Fault tolerance is another advantage of multipath routing. In single path routing fault tolerance is high because of single path between source and destination. The single paths are always failed due to congestion or any other reason. While routing redundant packets is not the only way to utilize multiple paths, it demonstrates how multipath routing can provide fault tolerance in the presence of route failures. To alleviate congestion as well as bottlenecks and maximize the resources for MANET, the ideal number of multipath routing should be taken into consideration [17].

\subsection{Problem and Proposed Solution}

The approach which is proposed in [13] on-demand based ad hoc routing protocol with multiple backup routes (ARMBR). In their ARMBR, Multiple backup paths are constructed, when the topology is changed, and the data are transmitted dynamically over the backup paths. The backup routes are generated in route data delivery phase. In this phase, a field HTD is inserted into the common header of the data packets. This field is used to determine the direction of the backup path. In route maintenance phase, the main route is repaired or invalid backup route is erased.

The following drawbacks are observed in this approach:

1. For forwarding packets this approach will take more time because it did not know whether the selected path is congested or not.

2. The delay is more in this approach because one path is failed it will select another path so the packet delay is increased.

In this paper, a congestion aware multipath routing protocol in mobile ad hoc networks (MANET) is proposed.

\section{RELATED WORKS}

An Ant Based Multipath Routing for Load Balancing and Congestion Control scheme is presented in [8]. Their multipath routing scheme has discovered the path when it is required at the start of a data session. Forward and backward ants are injected in the network so as to discover multiple paths to the destination. A reactive path is set up by the forward ant which provides the routing information of a node. The best path for each ant is selected based upon the number of hops and travel time.
Senthil Kumaran et al. [9] have proposed an early congestion detection and adaptive routing in MANET, which is described as EDAPR. At first, their EDAPR creates a NHN (noncongested neighbors) list. Then, it discovers a route to a destination by means of an NHN node. Queue status at node level is periodically computed by primary paths. The proposed early congestion detection technique recognizes congestion that is likely to happen and immediately it alerts NHN by transmitting warning messages. An adaptive path mechanism is used to find an alternate path.

A node-disjoint multipath routing method (NDMP-AODV) based on ad-hoc on-demand distance vector (AODV) routing protocol is put forwarded in [12]. Their approach has lessened the effect of link failure and they have also addressed the problem of frequent link failures. By getting the backup path stored in its routing table during the route discovery process, their NDMP-AODV guarantees that a node can transmit data after a route is broken without any delay.

Kun et al., [13] have proposed a new protocol, named ondemand based ad hoc routing protocol with multiple backup routes (ARMBR). It has improved existing on-demand routing protocols by constructing multiple backup routes, when the network topology changed. Their protocol could transmit data packets dynamically through backup routes. Also, they developed an analytic model to estimate the reconnection probability of their protocol. Their protocol is fully distributed and on-demand.

Congestion-aware routing protocol for mobile ad hoc networks is introduced in [14] by Xiaoqin Chen et al. Their technique is referred as CARM. Their technique has used a combined metric considering data-rate, channel delay, buffer delay, and retransmission count to oppose congestion and to enhance network utilization. In order to change the ad hoc networks robust and adaptive to congestion, the authors have utilized their combined metric along with the avoidance of mismatched link data-rate routes.

\section{PROPOSED MULTIPATH ROUTING PROTOCOL}

\subsection{Overview}

In this paper, first multiple paths are discovered using the ad hoc routing protocol with multiple backup routes (ARMBR) [13]. In this multipath routing, the bandwidth and end-to-end delay are considered. The delay includes weighted channel delay and buffer queuing delay. The paths which have better bandwidth will be initially selected. For selected paths, the congestion status of node is determined by using the early congestion detection technique which is based on the queue status and data arrival rate of the nodes.

If the congestion status is lower than the threshold value, the packets are directly sent through the path. If it is higher, a congestion aware routing is triggered. In congestion aware routing, routes having less end-to-end delay are selected.

\subsection{Multiple Route Discovery}

Starting of the route discovery, the sender sends the route request packet to all the neighbors. The neighbors will receive the packet and checks if it receives the same packet previously or not. If it got previously, just skip the packet otherwise it will forward to its neighbors. The packet format is 


\begin{tabular}{|l|l|l|l|l|}
\hline SID & DID & $\begin{array}{l}\text { Prev } \\
\text { Hop }\end{array}$ & $\begin{array}{l}\text { Neighbor } \\
\text { Hop }\end{array}$ & TTL \\
\hline
\end{tabular}

Fig. 1.Packet Format

In Fig (1), SID is the sender id, DID is the destination id, Prev Hop is the previous neighbor node and neighbor Hop is the next hop address and TTL is the time to live. When the neighbor node got the packet, then it will send to the neighbors. When they DID is matched then it will return to the source.

Routing tables are maintained to store the multiple paths. There are two types of tables maintained : one is main routing table (MRT) and the another one is multiple backup routes table (MBT). The MRT is used in the routing and it is a main table for routing. When the main route is not working then the MBT table can be used. The MBT table contains the more routes towards to the destination and if one route is not working then we will choose another one.

\subsubsection{Main Route Table (MRT)}

This table is mainly used for routing and this path is the best one among all the paths. The entries in the MRT are SID, DID, NH, Prev Hop, Next Hop, Status and TTL. SID is the source id, DID is the destination id, NH is the number of hops, Prev Hop is the previous hop of the route, Next Hop is the next hop of the route, Status is the node is available or not and TTL is the time to live. NH plays an important role in selecting the best path. The best path must have less number of numbers of hops. If the middle of the route, the node is with busy status then it will go to another route. If the route breaks then there is no need to go source, at which node the route breaks from there, it will take another route by using the MRT.

\begin{tabular}{|c|c|c|c|c|c|c|}
\hline SID & DID & NH & $\begin{array}{c}\text { Prev } \\
\text { Hop }\end{array}$ & $\begin{array}{c}\text { Next } \\
\text { Hop }\end{array}$ & Status & TTL \\
\hline$\ldots \ldots$ & $\ldots \ldots$ & 9 & $\ldots \ldots$ & $\ldots \ldots$ & Available & $\ldots \ldots$ \\
\hline$\ldots \ldots$ & $\ldots \ldots$ & 10 & $\ldots \ldots$ & $\ldots \ldots$ & Busy & $\ldots \ldots$ \\
\hline$\ldots \ldots$ & $\ldots \ldots$ & 12 & $\ldots \ldots$ & $\ldots \ldots$ & Available & $\ldots \ldots$ \\
\hline$\ldots \ldots$ & $\ldots \ldots$ & 18 & $\ldots \ldots$ & $\ldots \ldots$ & Busy & $\ldots \ldots$ \\
\hline
\end{tabular}

Fig 2: MRT table Format

The NH values are the integer and that is the count of hops between the source and destination. The Status is the two types' one is available, another one is busy.

\subsubsection{Multiple Backup Routes (MBT) Table}

MBT contains multiple backup routes. After selecting the paths into the MRT table, remaining paths are entered to the MBT table.

\begin{tabular}{|c|c|c|c|c|c|c|c|}
\hline SID & DID & $\mathrm{NH}$ & $\begin{array}{l}\text { Prev } \\
\text { Hop }\end{array}$ & $\begin{array}{l}\text { Next } \\
\text { Hop }\end{array}$ & Status & TTL & $\begin{array}{l}\text { Seq } \\
\text { No }\end{array}$ \\
\hline$\ldots \ldots$ & $\ldots \ldots$ & 9 & $\ldots \ldots$ & $\ldots .$. & Available & $\ldots \ldots$ & 1 \\
\hline$\ldots \ldots$ & $\ldots \ldots$ & 10 & $\ldots \ldots$ & $\ldots \ldots$ & Busy & ...... & 2 \\
\hline$\ldots \ldots$ & $\ldots \ldots$ & 12 & $\ldots \ldots$ & $\ldots \ldots$ & Available & $\ldots \ldots$ & 3 \\
\hline$\ldots \ldots$ & $\ldots \ldots$ & 18 & $\ldots \ldots$ & $\ldots \ldots$ & Busy & $\ldots \ldots$ & 4 \\
\hline
\end{tabular}

Fig 3: MBT table Format

The MBT contains the multiple routes and back route has seven fields. They are SID, DID, NH, Prev Hop, Next Hop, Status, TTL and Seq No. Seq No is the one more field that is added to the table and this is the sequence number of the route in that table.

\subsection{Selection of Routes}

In the selection of routes phase, the route which has maximum available bandwidth is selected as the primary route. Estimating available bandwidth allows a node to make an efficient decision before sending a frame in networks.

\subsubsection{Estimation of Availble Bandwidth}

Bandwidth [18] is the average number of packets serviced in each time unit between two nodes in the network. Whilee selecting the route, the nodes along the route have to be checkeed whether they are transmitting the packets or idle. At any specific instatnt of time, a link is either transmitting a packet at the full link capacity or it is idle. Idle period of channel is essential in calculation of bandwidth. The avialble Bandwidth is calculated using the following equation

$$
A B=\mathrm{B}_{\max } * \frac{\mathrm{T}_{\text {idle }}}{\mathrm{T}_{\text {interval }}}
$$

In (1), $A B$ is the available bandwidth, $B_{\max }$ is maximum bandwidth. $\mathrm{T}_{\text {idle }}$ is the idel time of the node and that is calucalte using this equation $T_{\text {idle }}=T_{\text {interval }}-T_{\text {busy }}$. $T_{\text {busy }}$ is the busy time of the node and this busy time the node will send the packets to the other nodes. In a period of time the node may change the state busy to idle, so the avialble bandwidth is updated for every $\mathrm{T}$ interval of time.

Among the established routes, the route with maximum available bandwidth will selected as the primary route. From the backup routes, the routes with less delay will be selected.

\subsubsection{Estimation of delay [14]}

Estimation of delay is done to select the routes from the backup routes. It involves channel waiting delay and the queuing delay.

$$
\mathrm{TD}=\mathrm{D}_{\mathrm{i}, \mathrm{j}}+\mathrm{Q}_{\text {delay }}
$$

In the equation (2), TD is the total delay, $\mathrm{D}_{\mathrm{i}, \mathrm{j}}$ is the channel waiting delay and $\mathrm{Q}_{\text {delay }}$ is the queuing delay.

\subsubsection{Channel waiting delay}

In the channel waiting delay the waiting delay in the channel due to overhead is calculated. Let the channel delay for the link $(i, j)$ between nodes $i$ and $j$ be defined as the interval between the start of the RTS transmission at node $i$ and the time the data packet is correctly received at node $\mathrm{j}$.

The channel delay is given by

$$
\mathrm{D}_{\mathrm{i}, \mathrm{j}}=\mathrm{T}_{\operatorname{minTCO}}+\mathrm{T}_{\mathrm{data}}
$$

In equation (3), $\mathrm{D}_{\mathrm{i}, \mathrm{j}}$ is the channel waiting time, $\mathrm{T}_{\text {minTCO }}$ is the time taken due to contention for the channel occupation due to MAC overhead and where $T_{\text {data }}=L_{\text {data }} / R_{i, j}$ is the data transmission time, $L_{d a t a}$ is the length of the data, and $R_{i, j}$ is the data-rate of the link. $\mathrm{T}_{\text {minTCO }}$ is calculated using the following equation

$$
\mathrm{T}_{\operatorname{minTCO}}=\mathrm{T}_{\operatorname{minCO}}+\mathrm{T}_{\mathrm{ac}}
$$

In equation (4), $T_{\operatorname{minCO}}$ is the minimum channel occupation due to MAC overhead and $\mathrm{T}_{\mathrm{ac}}$ is the is the time taken due to access contention (including NAV waiting and back-off intervals) and retransmissions. $\mathrm{T}_{\operatorname{minCO}}$ is calculated using the following equation

$$
\mathrm{T}_{\mathrm{minCO}}=\mathrm{T}_{\mathrm{RTS}}+\mathrm{T}_{\mathrm{CTS}}+3 \mathrm{~T}_{\mathrm{SIFS}}
$$


In equation (5), $\mathrm{T}_{\mathrm{RTS}}$ is the time to send request-to-send (RTS) packet, $\mathrm{T}_{\mathrm{CTS}}$ is the time to send clear-to-send (CTS) and $\mathrm{T}_{\mathrm{SIFS}}$ is the amount of time between the receipt of one packet and the transmission of the next is called a short interframe space (SIFS). The queuing delay is calculated after finding the channel waiting delay.

\subsubsection{Queuing Delay}

Queuing delay is very useful in measuring of congestion. Queuing delay is the delay time it is waiting in the queue. The number of packets that are buffered in the node at time $t$ is called as queuing delay. The delay in the interface queue of an intermediate node is used to select the maximum throughput paths, avoiding the most congested links. The queuing delay is calculated using the following equation

$$
\mathrm{Q}_{\text {delay }}=\mathrm{Q}_{\mathrm{i}, \mathrm{k}} / \mathrm{t}
$$

In equation (6), $Q_{i, k}$ is the number of packets buffered in the node $\mathrm{i}$ queue bound for node $\mathrm{k}$ at time $\mathrm{t}$. $\mathrm{K}$ is the queue bound of the node. The queue length [19] is estimated by the following equation

$$
Q L=\alpha * Q L_{\text {old }}+(1-\alpha) * \mathrm{QL}_{\text {current }}
$$

In equation (7), $\mathrm{QL}$ is the average queue length and $\mathrm{QL}_{\text {current }}$ is the current queue length. $\alpha$ is constant and that can be any number selected from the range $[0,1]$. QL indicate the nodes traffic load. Arrival rate [20] can be calculated using the following equation

$$
A R=\lim _{\mathrm{t} \rightarrow \infty} \frac{\mathrm{N}_{\mathrm{p}}(t)}{\mathrm{t}}
$$

In equation (8), $\mathrm{AR}$ is the arrival rate and $\mathrm{N}_{\mathrm{p}}$ is the number of packets that are arriving in a tiem $t$.

\subsection{Congestion Estimation}

In the congestion estimation phase, the congestion is estimated based on the queue length and arrival rate. If the queue length and arrival rates are low, then the congestion will be less otherwise the congestion will be more. The channel delay and queuing delay are used here since they are good measurement of congestion with combination of access contention, channel occupation, and data-rate. The congestion is estimated using the following algorithm

$\begin{aligned} & \text { 1. Start } \\ & \text { 2. } \\ & \text { 3. } \\ & \text { equation (8)) } \\ & \text { 4. } \\ & \text { length } \\ & \text { 5. }\end{aligned}$
$\mathrm{AR}=$ Arrival Rate
$\mathrm{Th} 1=$ threshold value of queue
$\mathrm{Th} 2=$ threshold value of arrival
rate

6.

7. Select the primary path based on available bandwidth

8.

9. If $((\mathrm{QL}<\mathrm{Th} 1)$ and $(\mathrm{AR}<\mathrm{TH} 2))$

10. Congestion Status $=$ low

11. Else

12. Congestion status $=$ high

14. If (congestion status $==$ high $)$

$15 . \quad\{$

16.

the source
17.

18.

19.

20.

21.

22.

23.

24.

delay

25.

26. node . count $=$ node. count +1 ;

If (node.count >= 3 )

Congestion aware routing $=$ start

If $($ congestion aware routing $=$ start $)$

Take a path from MBT table which has less

End

\section{Algorithm for Congestion estimation}

Here, first the primary path is selected for the MRT table. Each node Ni checks the queue length and data arrival rate based on which the congestion status is determined. Two threshold values are defined for queue length and arrival rate. If the queue length and arrival rate are less than these threshold values then the congestion will be less, otherwise it will be considered as high.

When the congestion level is high then an early congestion warning is sent to the source from $\mathrm{Ni}$. If source receives a congestion warning message from more than one node along the path, Congestion aware routing is triggered. In congestion aware routing, an alternate path from the least delay backup path list will be selected and transmission is rerouted along the path.

\subsection{Congestion aware Multipath Routing}

In the congestion aware multipath routing protocol, first the multiple route discovery phase is used. In this phase, multiple routes are selected and stored into two tables: MRT and MBT. MRT is the main table from which the primary route is selected. MBT is the backup table.

The delay of the all the nodes are calculated and when the primary route fails, the route with less delay will be selected. Among the established routes, the route with maximum available bandwidth will selected as the primary route. From the backup routes, the routes with less delay will be selected.

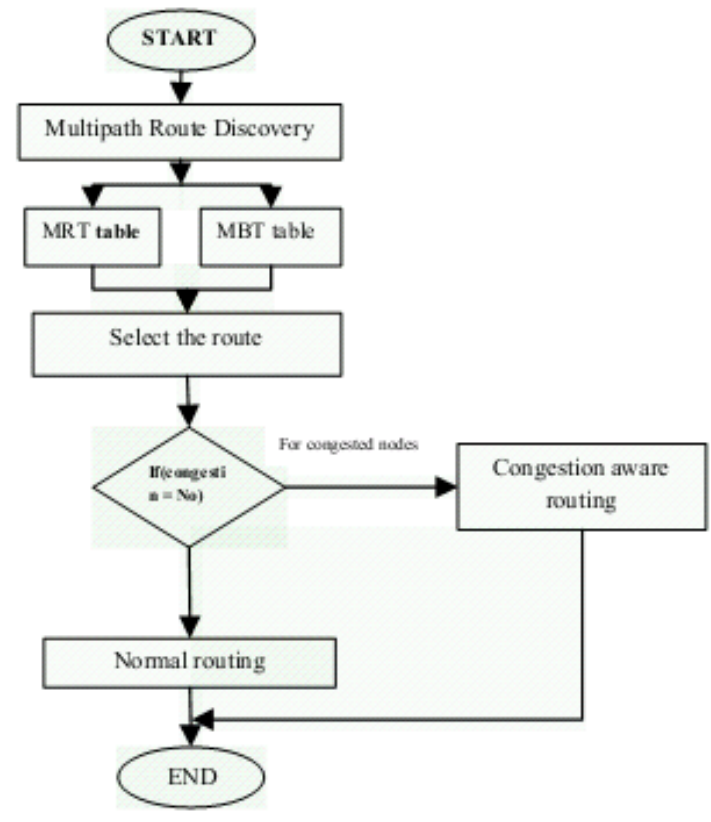

Fig 4: Total process flow 
The congestion status is checked based on the queue length and arrival rate. If the congestion status is high then an early congestion warning is sent to the source. If source receives a congestion warning message from more than one node along the path, congestion aware routing is triggered.

The congestion aware routing will start when the source gets the congestion warning messages. In congestion aware routing, an alternate path from the least delay backup path list will be selected and transmission is rerouted along the path.

Consider the below example. $\mathrm{S}$ is the sender and $\mathrm{D}$ is the destination. $\mathrm{S}$ want to send the packets to the $\mathrm{D}$ and $\mathrm{S}$ sends RREQ packets to all neighbouring nodes. When the packet reach the destination, then the source gets replay packets. Source will save the all possible paths in MRT and MBT tables. All possible paths from $\mathrm{S}$ to $\mathrm{D}$ are

$$
\begin{aligned}
& \mathrm{S} \rightarrow \mathrm{X} \rightarrow \mathrm{V} \rightarrow \mathrm{P} \rightarrow \mathrm{D} \\
& \mathrm{S} \rightarrow \mathrm{X} \rightarrow \mathrm{O} \rightarrow \mathrm{P} \rightarrow \mathrm{D} \\
& \mathrm{S} \rightarrow \mathrm{N} \rightarrow \mathrm{M} \rightarrow \mathrm{D} \\
& \mathrm{S} \rightarrow \mathrm{B} \rightarrow \mathrm{Y} \rightarrow \mathrm{U} \rightarrow \mathrm{D}
\end{aligned}
$$

Those are all multipaths for send the packets. The primary path which has less number of hops which is saved in MRT table and remaining are saved in MBT table.

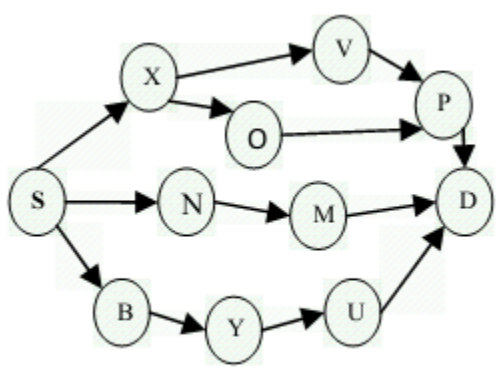

Multipath Route Selection

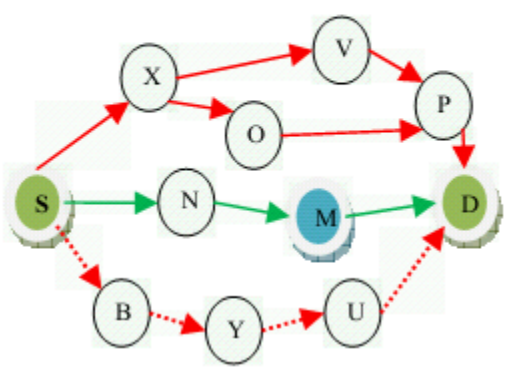

Congestion Aware

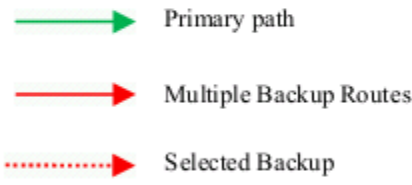

Congested

Fig 5: Congestion Aware Routing

The primary path $\mathrm{S} \rightarrow \mathrm{N} \rightarrow \mathrm{M} \rightarrow \mathrm{D}$ is selected because it has less number of hops. When the source gets the congestion warning messages from the node $\mathrm{M}$ then, the congestion aware routing will be started. In the congestion aware routing, the backup route $\mathrm{S} \rightarrow \mathrm{B} \rightarrow \mathrm{Y} \rightarrow \mathrm{U} \rightarrow \mathrm{D}$ is selected from the MBT table since it hasless delay compared with others. 


\section{SIMULATION RESULTS}

\subsection{Simulation Model and Parameters}

NS-2 [18] is used to simulate the proposed Multipath routing for Reducing Congestion and Delay (MPRCD) technique. In the simulation, the channel capacity of mobile hosts is set to the same value: 2 Mbps. The distributed coordination function (DCF) of IEEE 802.11 is used for wireless LANs as the MAC layer protocol. It has the functionality to notify the network layer about link breakage.

In simulation, 100 mobile nodes move in a 1000 meter x 1000 meter square region for 100 seconds of simulation time. It is assumed that each node moves independently with the same average speed. All nodes have the same transmission range of 250 meters. In the simulation, the speed varied as $2,4,6,8$ and $10 \mathrm{~m} / \mathrm{s}$. The simulated traffic is Constant Bit Rate (CBR).

The simulation settings and parameters are summarized in table

Table1. Simulation Parameters

\begin{tabular}{|l|l|}
\hline No. of Nodes & 100 \\
\hline Area Size & $1000 \mathrm{X} 1000$ \\
\hline Mac & 802.11 \\
\hline Radio Range & $250 \mathrm{~m}$ \\
\hline Simulation Time & $100 \mathrm{sec}$ \\
\hline Traffic Source & CBR \\
\hline Packet Size & 512 \\
\hline Mobility Model & Random Way Point \\
\hline Speed & $2,4,6,8$ and $10 \mathrm{~m} / \mathrm{s}$ \\
\hline Pause time & $5 \mathrm{~s}$ \\
\hline Rate & $50,100,150,200$ and \\
& $250 \mathrm{~Kb}$ \\
\hline
\end{tabular}

\subsection{Performance Metrics}

The MPRCD technique is compared with the ARMBR [13] protocol. The performance is evaluated mainly according to the following metrics:

Average end-to-end delay: The end-to-end-delay is averaged over all surviving data packets from the sources to the destinations.

Average Packet Delivery Ratio: It is the ratio of the No. of packets received successfully and the total no. of packets sent.

Overhead: It is the number of error control packets found during the data transmission.

\subsection{Results}

\section{A. Based on Rate}

In the first experiment, the transmission rate is varied as $50,100,150,200$ and $250 \mathrm{~Kb}$.

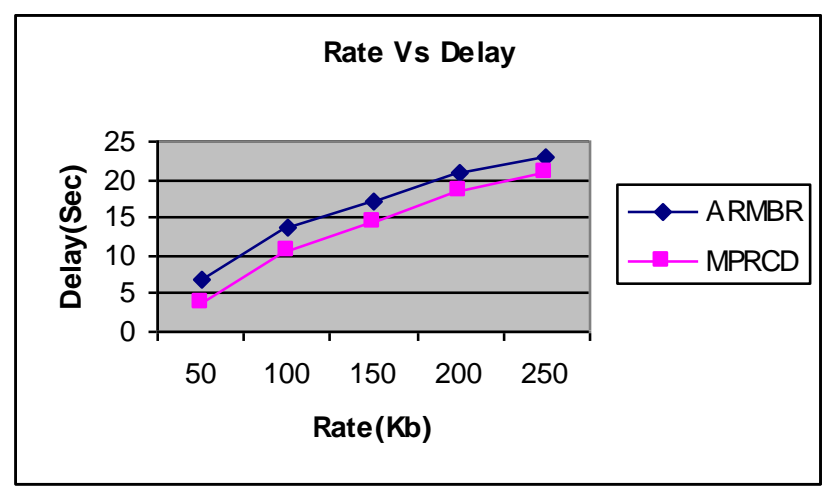

Fig 6: Rate Vs Delay

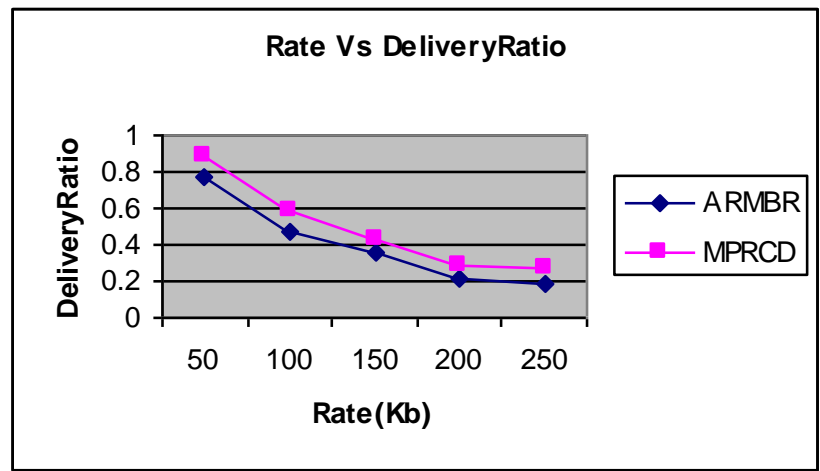

Fig 7: Rate Vs Delivery Ratio

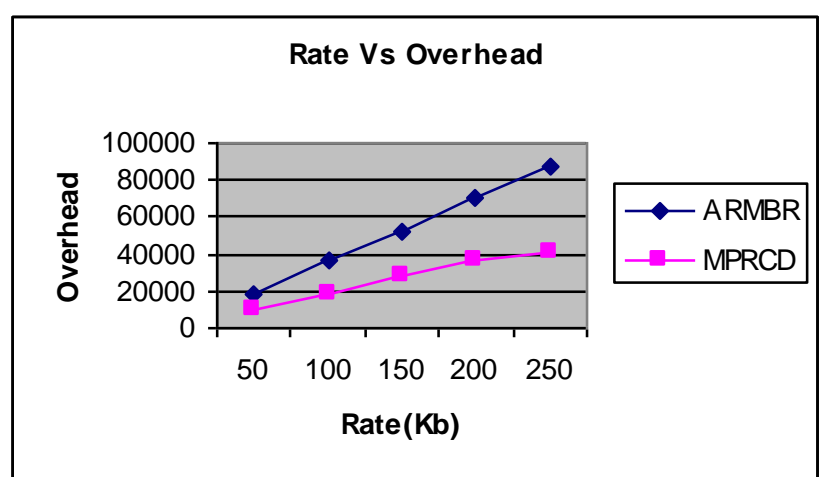

Fig 8: Rate Vs Overhead

From Fig 6, it can be seen that the delay of proposed MPRCD is less than the existing ARMBR protocol.

From Fig 7, it can be seen that the delaivery ratio of proposed MPRCD is higher than the existing ARMBR protocol.

From Fig 8, it can be seen that the overhead of proposed MPRCD is less than the existing ARMBR protocol.

B. Based on Speed

In the second experiment, the node speed is varied as $2,4,6,8$ and $10 \mathrm{~m} / \mathrm{s}$. 


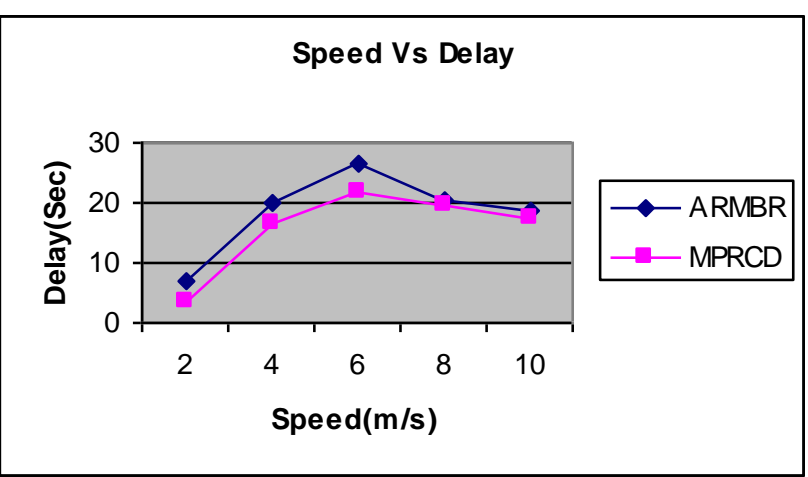

Fig 9: Speed Vs Delay

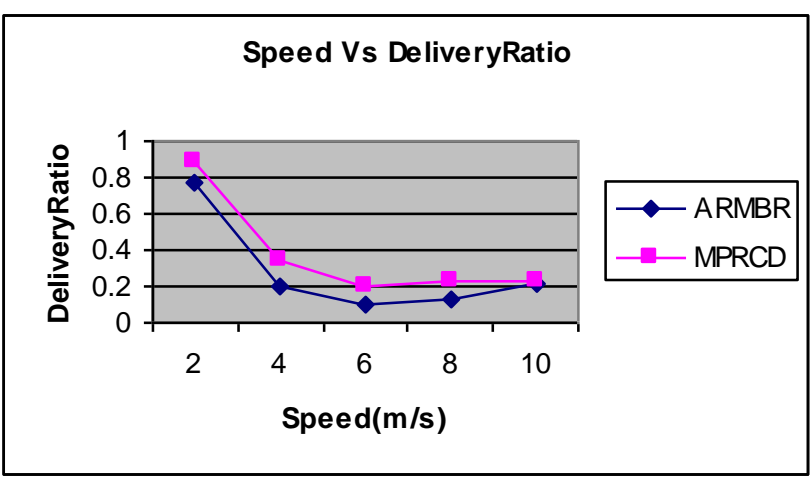

Fig 10: Speed Vs Delivery Ratio

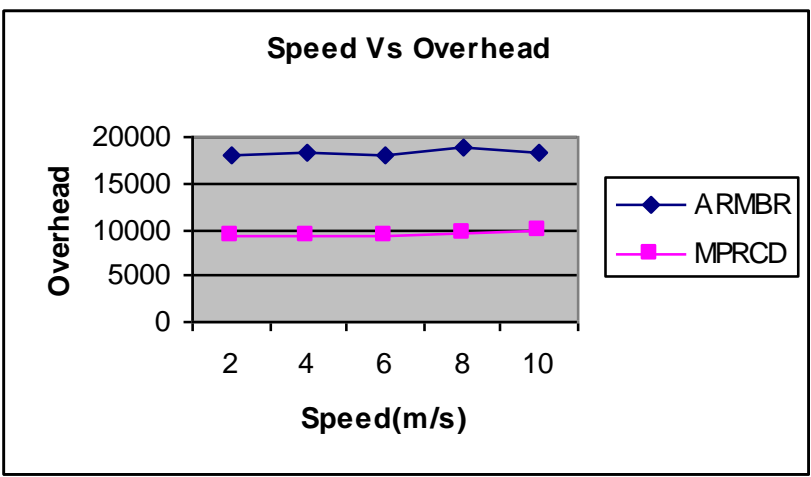

Fig 11: Speed Vs Overhead

From Fig 9, it can be seen that the delay of proposed MPRCD is less than the existing ARMBR protocol.

From Fig 10, it can be seen that the delaivery ratio of proposed MPRCD is higher than the existing ARMBR protocol.

From Fig 11, it can be seen that the overhead of proposed MPRCD is less than the existing ARMBR protocol

\section{CONCLUSION}

In this paper, a congestion aware multipath routing protocol for MANET is proposed. The paths which have better bandwidth will be initially selected. For selected paths, the congestion status of node is determined using the early congestion detection technique which is based on the queue status and data arrival rate of the nodes. If the congestion status is lower than the threshold value, the packets are directly sent through the path. If it is more than the threshold value, a congestion aware routing is initiated. In congestion aware routing, routes having less end-to-end delay are selected to lower the congestion. Using this protocol, routes with mismatched link data-rates can be prevented. By simulation results, it is proved that the packet delivery ratio is improved and the end to end delay is reduced.

\section{REFERENCES}

[1] Fang Liu, Kai Xing, Xiuzhen Cheng and Shmuel Rotenstreich, "Energy proefficient MAC layer protocols in ad hoc networks", Resource Management in Wireless Networking.

[2] Jyoti Jain, Mehajabeen Fatima, Dr. Roopam Gupta and Dr. .K.Bandhopadhyay, "OVERVIEW AND CHALLENGES OF ROUTING PROTOCOL AND MAC LAYER IN MOBILE AD-HOC NETWORK", Journal of Theoretical and Applied Information Technology, 2009.

[3] A.Rajaram and Dr. S. Palaniswami, "The Trust-Based MAC-Layer Security Protocol for Mobile Ad hoc Networks", (IJCSE) International Journal on Computer Science and Engineering, Vol. 02, No. 02, 2010.

[4] H. Dong, I. D. Chakares, A. Gersho, E. Belding-Royer $\dagger$, J. D. Gibson, "Selective Bit-error Checking at the MAC Layer for Voice Over Mobile Ad Hoc Networks with IEEE 802.11".

[5] Imrich Chlamtac, Marco Conti and Jennifer J.-N. Liu, "Mobile ad hoc networking: imperatives and challenges", Elsevier, 2003.

[6] Prof. S.A. Jain, Mr. Abhishek Bande, Mr. Gaurav Deshmukh, Mr. Yogesh Rade and Mr. Mahesh Sandhanshiv, " An Improvement In Congestion Control Using Multipath Routing In Manet”, International Journal of Engineering Research and Applications (IJERA), Vol. 2, Issue 3, May-Jun 2012.

[7] Mandeep Kaur Gulati and Krishan Kumar, "Survey of Multipath QoS Routing Protocols For Mobile Ad Hoc Networks", International Journal of Advances in Engineering \& Technology, May 2012.

[8] S. Soundararajan and R. S. Bhuvaneswaran, "Ant Based Multi-path Routing for Load Balancing and Congestion Control in MANETs", Journal of Information \& Computational Science, 2012.

[9] T. Senthil Kumaran and V. Sankaranarayanan, "Early congestion detection and adaptive routing in MANET", Egyptian Informatics Journal, 2011. 
[10] Shitalkumar Jain, Shrikant Kokate, Pranita Thakur and Shubhangi Takalkar, "A Study of Congestion Aware Adaptive Routing Protocols in MANET", Computer Engineering and Intelligent Systems, Vol 3, No.4, 2012.

[11] K.Vats, Indu Vishnoi and Anju Shukla, "NOVEL ARCHITECTURE OF DELAY AND ROUTING IN MANET FOR QoS", International Journal of Engineering Science and Technology (IJEST), Vol. 3 No. 1 Jan 2011.

[12] Chhagan Lal ,V.Laxmi and M.S.Gaur, "A Node-Disjoint Multipath Routing Method based on AODV protocol for MANETs", 26th IEEE International Conference on Advanced Information Networking and Applications, 2012.

[13] 13 Kun-Ming Yu, Chang Wu Yu and shi-Feng Yan, “An Ad hoc Routing Protocol With Multiple Backup Routes", Springer, Wireless Personal Communications, vol. 57, no. 4, pp. 533-551, 2011.

[14] Xiaoqin Chen, Haley M. Jones and A .D .S. Jayalath, "Congestion-Aware Routing Protocol for Mobile Ad Hoc Networks", IEEE, 2007.
[15] R. R. Chen and K. Khorasani, "Markovian Jump Guaranteed Cost Congestion Control Strategy for Mobile Networks Subject to Differentiated Services Traffic", 50th IEEE Conference on Decision and Control and European Control Conference (CDC-ECC), 2011.

[16] Christos Tachtatzis and David Harle, "Performance Evaluation of Multi-path and Single-path Routing Protocols for Mobile Ad-Hoc Networks”, IEEE, 2008.

[17] Stephen Mueller, Rose P. Tsang and Dipak Ghosal, "Multipath Routing in Mobile Ad Hoc Networks: Issues and Challenges", 2004.

[18] Fujian Qin, and Youyuan Liu, "Multipath Routing forMobile Ad Hoc Network", International Symposium on Information Processing, 2009.

[19] Xuemei Gao, Xinming Zhang, Dong Shi, Fengfu Zou and Wenbo Zhu, "Contention and Queue-aware Routing Protocol for Mobile Ad h oc Networks”, IEEE, 2007.

[20] Ming Zhao and Wenye Wang, "Analyzing Topology Dynamics in Ad Hoc Networks Using A Smooth Mobility Model",

IEEE,

2007 